2010-08-11

\title{
A Monocular Marker-Free Gait Measurement System
}

\author{
Jane Courtney \\ Technological University Dublin, jane.courtney@tudublin.ie \\ Annraoi M. De Paor \\ University College Dublin, annraoi.depaor@ucd.ie
}

Follow this and additional works at: https://arrow.tudublin.ie/biodevart

Part of the Analytical, Diagnostic and Therapeutic Techniques and Equipment Commons, Biomedical Commons, Biomedical Engineering and Bioengineering Commons, Rehabilitation and Therapy Commons, and the Sports Sciences Commons

\section{Recommended Citation}

Courtney, J., De Paor, A. (2010) A Monocular Marker-Free Gait Measurement System. IEEE Transactions on Neural Systems and Rehabilitation Engineering. Vol. 18 no. 4 pp. 453 - 460 ISSN: 1534-4320. doi:10.1109/TNSRE.2010.2041792

This Article is brought to you for free and open access by the Biomedical Devices and Assistive Technology Research Group at ARROW@TU Dublin. It has been accepted for inclusion in Articles by an authorized administrator of ARROW@TU Dublin. For more information, please contact arrow.admin@tudublin.ie, aisling.coyne@tudublin.ie,gerard.connolly@tudublin.ie.

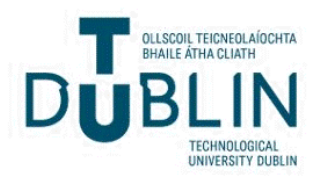




\title{
A Monocular Marker-Free Gait Measurement System
}

\author{
Jane Courtney, Member, IEEE, and A. M. de Paor
}

\begin{abstract}
This paper presents a new, user-friendly, portable motion capture and gait analysis system for capturing and analyzing human gait, designed as a telemedicine tool to monitor remotely the progress of patients through treatment. The system requires minimal user input and simple single-camera filming (which can be acquired from a basic webcam) making it very accessible to nontechnical, nonclinical personnel. This system can allow gait studies to acquire a much larger data set and allow trained gait analysts to focus their skills on the interpretation phase of gait analysis. The design uses a novel motion capture method derived from spatiotemporal segmentation and model-based tracking. Testing is performed on four monocular, sagittal-view, sample gait videos. Results of modeling, tracking, and analysis stages are presented with standard gait graphs and parameters compared to manually acquired data.
\end{abstract}

Index Terms-Automated tracking, gait analysis, human movement, motion analysis.

\section{INTRODUCTION}

\section{A. Motivation}

C URRENT gait measurement methods involve complex marker systems, multiple cameras, a dedicated gait laboratory and trained personnel. This paper presents a simple single-camera system which has low processing time and is usable remote from the filming location and without the need for qualified gait analysts at the data acquisition stage. While this system is not intended to replace marker-based systems, it allows the study of gait to broaden beyond the gait laboratory while providing results comparable to those achievable with standard systems. It has been used, for example, to analyze gait in video clips filmed in another country. It is hoped that its simplicity will encourage therapists and patients to participate in gait studies and make the most of the technology available.

\section{B. Marker-Based Systems}

Marker-based systems are still the most reliable and widely used. However, they require specific equipment and expertise, not accessible outside a gait laboratory. This can be a significant issue when patients are too unwell to travel or when large data sets are required for study. Also, as demonstrated in [1], gait facilities are not readily available to many potential users.

Within a gait laboratory, marker placement is still difficult. Marker positions have a significant effect on system output.

Manuscript received October 07, 2008; revised May 27, 2009; accepted December 14, 2009. First published February 08, 2010; current version published August 11, 2010.

J. Courtney is with Electrical Engineering Department, Dublin Institute of Technology, Dublin 6, Ireland (e-mail: jane.courtney@dit.ie).

A. M. de Paor is with the Department of Electrical Engineering, National University of Ireland, Dublin 2, Ireland.

Color versions of one or more of the figures in this paper are available online at http://ieeexplore.ieee.org.

Digital Object Identifier 10.1109/TNSRE.2010.2041792
Slight inaccuracies, particularly around the joints, can cause failure. Reliability can be improved, though this requires added cameras, added expense and restriction to a fixed filming location. Passive marker systems are less intrusive than active but require more markers to compensate for vulnerability to occlusion [2]. This happens during walking as the subject's arms swing back and forth, occluding the pelvic region. Many studies on trends in gait analysis, e.g., [3], have predicted that future developments in gait analysis will tend away from marker-base systems.

\section{Marker-Free Gait Analysis}

Many research groups are striving to develop the first fully automated marker-free gait analysis system. There are already some commercially available marker-free motion capture systems, e.g., [4]. To date however, none is completely automated and all require a gait laboratory environment, several measurements of the subject and/or manual intervention at various stages. These systems sometimes suffice, but have not been readily embraced by therapists as an alternative to marker-based systems in monitoring pathological gait. Some portable gait measurement systems are available, e.g., [5] and [6], but these concentrate on velocity measurements and do not acquire full kinematics. Here, this task is tackled using computer vision based techniques.

The greatest challenge for computer vision lies in analysis of the lower limbs in the sagittal plane. The difficulties in the sagittal plane stem from the similarity and proximity of the two legs and from speed change during the swing phase. Many attempts at marker-free systems have been based on feature detection and tracking [7] or on apparent motion [8]. However, on crossover of the legs, during swing, image features become less well defined and it is difficult to identify any apparent motion. Although this is not technically occlusion, the result is the same: tracking cues are lost. As the legs cross, the image of the moving leg becomes blurred and indistinguishable from the stationary leg. With standard techniques, this can lead to motion vectors having erroneous zero values. Marker-free techniques are still being investigated in this area. Review paper [9] looks at the various tasks involved in motion analysis of the human body from a computer vision perspective and discusses recognition of human activities from image sequences. Survey [10] presents recent developments, focusing on whole-body motion and discussing various methodologies. These reviews should be consulted for a thorough summary of current research.

\section{Design Goals}

With patients and therapists in mind, the following goals were set for this design.

- The system must be completely automated. 
- The system must be simple to use, requiring minimal training.

- The output will be a comprehensive set of sagittal plane gait graphs and parameters, sufficiently accurate for clinical diagnosis.

- The input will be a single video file of the patient walking.

- The gait video can be filmed in any reasonable environment without significant restrictions.

- The subject can be fully and appropriately clothed.

- The subject can walk freely.

While accuracy is important, this design is intended as a firststop gait measurement system and is not intended, at this stage, to compete with marker-based systems in terms of accuracy. In addition, because of the specific application, expectations are restricted to the following.

- It is reasonable to expect adequate lighting and contrast in the filming environment.

- The data will be filmed from a stationary camera.

- The subject will walk approximately fronto-parallel to the camera.

- The subject will be fully visible in all frames from head to toe.

- Clothing will not hide the subject's leg outline, for example, skirts may not be worn.

- The height of the subject is known.

An initial design attempt was made previously but the difficulty at leg crossover could not be overcome and the untracked leg had to be manually removed from each frame [11]. Since then, a method based on spatiotemporal image analysis has been developed which outlines the tracked leg in each frame, distinguishing it from the untracked leg, thus overcoming the problem. This results in fully automated tracking and a robust gait measurement system.

\section{Motion TRACKING}

Because this system focuses solely on the motion of the lower limbs, it is helpful to use known information in the design. This reduces the ability to measure alternative movements, but allows us to achieve the greatest accuracy and reliability for our particular application.

Model-based methods can be divided into appearance modeling and motion modeling. The former allows us to interpret the image of the human body as a set of connected moving objects. Each limb segment can be tracked either dependent on the motion of its connected segments or completely independently, allowing a considerable amount of freedom of motion. Motion modeling allows us to restrict behavior to known or reasonable movements and can be used to make good predictions when image measurements become unreliable. This can be particularly useful when measuring a defined motion.

The main advantage of model-based methods is that they are reasonably insensitive to the problems that cause other methods to fail-image noise, occlusion, lost edge cues, lack of image features, varying appearance, etc. In a simple example, if the thigh is approximated as a rectangle in a sagittal plane view and the arm swings in front of it, thereby occluding one of the edges, the correct position will still give the best fit result as most of the other three sides are still visible. Modeling methods can be combined with direct template matching techniques, e.g., correlation matching, to take advantage of the best available techniques.

By knowing about the shape of the object and its motion, the system can decide which type of model to use at each stage. Visual measurements such as edges and textures can be trusted when the leg is in stance so the structural model can be used. However, in swing, the image of the leg becomes blurred, edges are lost, and textures vary, so motion predictions are used for greater reliability. While model-based methods can be very helpful in interpreting image data and restricting the search space, it is important not to excessively constrain the motion. The system described here is aimed at measuring pathological gait, so it is important that model-based methods are not restricted to normal gait. Similarly, the structural or appearance-based model must not exclude limb deformations and artificial limbs.

In many motion systems, hidden Markov models (HMMs), or equivalent sorting systems, are used to attach the gathered data to predefined poses or motion models, e.g., [12] and [13]. These approximate the motion well and can be used to reconstruct a video scene or identify a person by gait. They are not, however, direct measurements and so not accurate enough to pick up on the nuances of pathologies. On the other hand, if no restrictions to motion interpretation are applied at all, nonsensical results can occur, such as unnatural joint angles and detachment of limbs.

Without the addition of model-based tracking, energy minimization techniques stray when the energy cues (edges, corners, or textures) are weak or transient as is often the case in lower limb movement. When mistakes are made, such systems go astray and cannot recover easily. In the CONDENSATION algorithm devised by Blake and Isard [14], a more robust method is presented for tracking. However, it requires all possible states to be retained at each stage, thereby decreasing efficiency and computational speed. Also, as revealed by Deutscher et al. [15], it still strays easily and takes several frames to recover.

In designing a model-based system, it is important to decide which restrictions are universally applicable and which are overly constricting and exclusive of certain pathologies. In this design, model restrictions have been kept to a minimum. In order to allow for variations in body shape due to deformations or artificial limbs, a basic structural model is used that simply represents the position and orientation of the limb segments at each point in time, remaining reasonably independent of dimensions or actual shape.

\section{SYSTEM DESIGN}

If the region of interest (in this case, the tracked leg) is identified, the task reduces to a shape-fitting problem. The approach taken here is divided into two parts: model fitting and segmentation.

\section{A. Model Fitting}

The model used here is an ellipse-based hierarchical tree structure. The body is divided into segments of coherent motion from the head down, each segment dependent on the position and orientation of the previous one. For example, once the 
thigh is located and oriented, this restricts possible positions and orientations of the shank and so on. Then, each segment is represented by a shape containing information about position and orientation at each time frame. From sequential positions and orientations, motion vectors are deduced.

Ellipses were found to be the best choice for all body segments. They are similar in shape to the segments of interest and have well-defined positions and orientations. Also, they are easily attached directly to segment outlines, making them fast and efficient to use. A novel ellipse-fitting algorithm [16] was devised for this task.

\section{B. Segmentation}

In fitting the model, it is assumed that the region being modeled is clearly defined and outlined. Therefore, a method of segmenting the region being modeled from the rest of the image must be designed. Many moving images can be segmented into areas of coherent motion and these modeled as independently moving objects. However, human bodies consist of parts that are not only similar in appearance but also similar in movement and position, making them difficult to separate. In addition, individual segments do not move independently but are connected and affect each other's motion. This problem is most exaggerated in the lower limbs where the moving parts on the leg being tracked have counterparts on the other leg. The two legs are generally very similar in appearance and texture and so there may be little or no apparent change in the image as they move in front of one another. Particularly at crossover, the outline can be blurred and significant edges lost. At this point, model-fitting methods can give erroneous results, which propagate through the image sequence. A segmentation method is needed which can recover from erroneous matches and can approximate the position of the outline when edge cues are lost.

Many methods were tested for achieving this segmentation, including optical flow vectors, clustering methods and energy functions cued by segment features. None of these were reliable enough over long video sequences or from a monocular viewpoint and few were able to recover from straying. However, a method presented by Nyogi and Adelson [17] examines the video sequence in a novel way that allows the outline to be tracked robustly. In this method, spatiotemporal images are analyzed to track the outline of the body. Gait recognition results were presented showing the success of the method in tracking human gait. However, for full gait measurement, the algorithm proved sensitive to some parameter values and, though the outline was quite robustly traced, when it did stray, the gait data were badly affected. This method has been extended here to make it fully automatic and reliable enough for gait measurement with the introduction of some automated measurement techniques outlined in Section IV. In addition, the segmentation technique is coupled with the model-based tracking, using our fast ellipse-fitting method, to generated reliable gait data.

\section{IMPLEMENTATION}

\section{A. The xyt Block}

In the spatiotemporal image analysis method, video frames from a stationary camera are stacked to create a 3-D block with

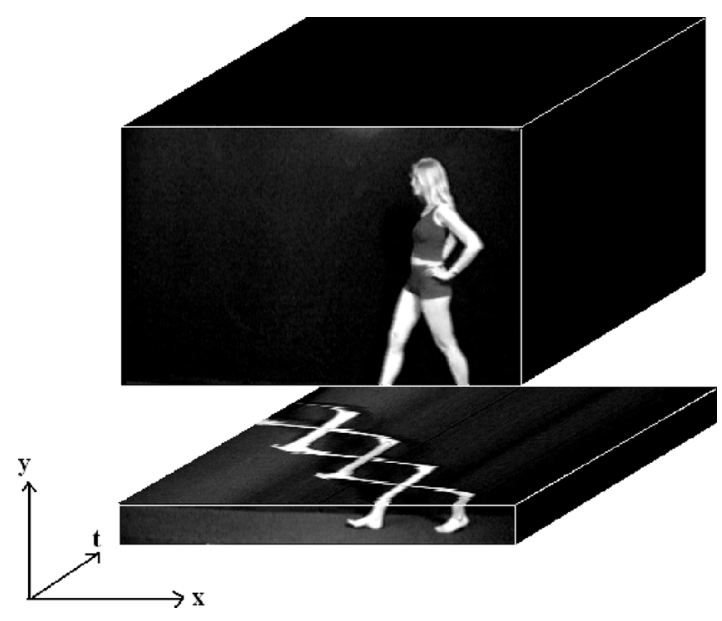

Fig. 1. The xyt block for the gait laboratory sequence.

two of its dimensions representing horizontal and vertical directions and the third representing time (see Fig. 1). An image of the movement in the video is obtained by slicing the block in the $\mathrm{xt}$ direction. This image is particularly useful for recognizing and analyzing walking because of an interesting characteristic of leg motion. In the case of a sagittal view of a human walking in fronto-parallel to a stationary camera, a distinct, braided pattern is observed in slices around the leg. The braids are formed by periodic motion of the legs through swing and stance. While the legs appear close to each other in the XY plane, causing occlusion and interference, in the xt plane they are clearly separate. If these two patterns can be outlined separately in the slice, the two legs would be distinct from one another throughout the video sequence. In order to automate the initialization of this outlining process, the parameters that define the braided shape must first be identified.

\section{B. Walking Path}

The walking path shows the subject's progress through the video sequence. This is used to guide contour initialization, calculate the bounding rectangle and determine the velocity of the walker. In order to determine the walking path of the subject, the xyt block is collapsed into a single image $W$. The intensity of the pixel at location $\left(x_{i}, t_{i}\right)$ in this image is given by

$$
W\left(x_{i}, t_{i}\right)=\frac{1}{h} \sum_{y=1}^{h} I\left(x_{i}, y, t_{i}\right)
$$

where $h$ is the height of the block (the same as the height of the video frames) and $I\left(x_{i}, y, t_{i}\right)$ is the intensity at point $\left(x_{i}, t_{i}\right)$.

This image is thresholded to highlight the region of most activity. By gathering all points from the diagonal pattern image $W$, a simple least-squares minimization (LSM) algorithm is used to find the parameters of the line $t=m x+c$ which defines the path of the walker. While a similar method is used in [18] for finding human walkers, the replacement of LSM for the Hough Transform here increases the speed of computation and gives a more exact result for the case of a single walker. Using these parameter values, the velocity of the walker is calculated. The frame rate of the video, $f$ and the projection ratio, $R$ is approximated. In a complete $3-\mathrm{D}$ system, the projection matrix could be 


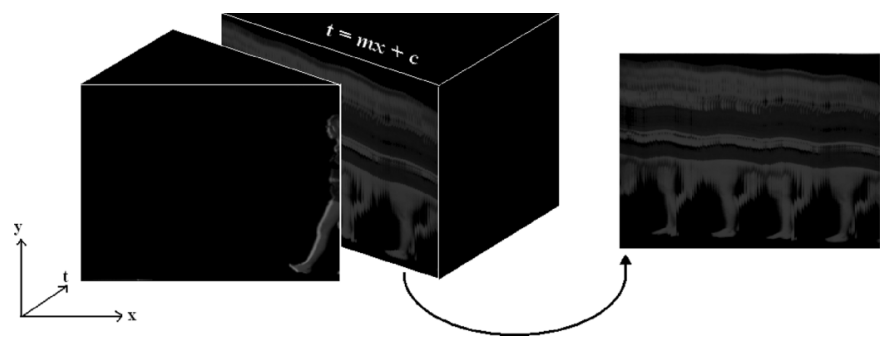

Fig. 2. The xyt block sliced along the path of the walker, $t=m x+c$, and the image acquired from this slice.

used to give more accurate results for $R$ but because the camera is stationary and the subject keeps at a reasonably constant far distance from it, comparing the subject's height in centimeters to their height in pixels in the video frame gives a good approximation of the ratio in pixels per centimeter. The velocity is then calculated as

$$
v=\cdot \frac{1}{m} \cdot \frac{f}{R} \quad\left[\frac{\mathrm{cm}}{\mathrm{s}}\right] .
$$

\section{Bounding Rectangle}

The bounding rectangle is the approximate rectangular area occupied by the subject in each video frame. Determining the bounding rectangle minimizes further calculations, gives limits to the outlining algorithm and helps divide the body into segments.

The width and horizontal position of the rectangle change as the walker moves across the camera plane so, while an average value for the head and toe heights, $y_{h}$ and $y_{t}$, will suffice, a sequence of horizontal bounds, $l(\mathrm{t})$ and $r(\mathrm{t})$, are required. In order to identify $y_{h}$ and $y_{t}$, the walking path of the subject is first determined. By slicing the block along this path, the image shown in Fig. 2 is obtained. Here it is seen that the vertical bounds of the subject change slightly along the path so average values for these bounding heights must be determined.

First, the stance positions are identified. This is done by calculating the projection of the intensity on the horizontal axis and examining it for peaks. Next, the vertical projection is calculated, projecting only the columns at the peaks, since these are the only locations where the subject is visible along their entire height. Horizontal projection $H(x)$ and vertical projection $V(y)$ is seen in Fig. 3.

From $V(y)$, the mean vertical projection, $V$ is calculated and mean-crossing points identified. The lowest crossing point is taken as the toe height, $y_{t}$ and highest as the head height, $y_{h}$. Having determined the vertical bounds, the height of the subject is segmented into approximations of individual body segments by using reasonable average body ratios. Ratios were tested from studies of the human body in medicine, art, and computer vision to choose reasonable approximations for the joint locations.

To determine horizontal bounds, the horizontal projection of each frame is examined. Sample bounding rectangles including body segmentations are shown in Fig. 4. The same vertical bounds and joint approximations are used throughout the sequence so in some frames they are slightly higher or lower than

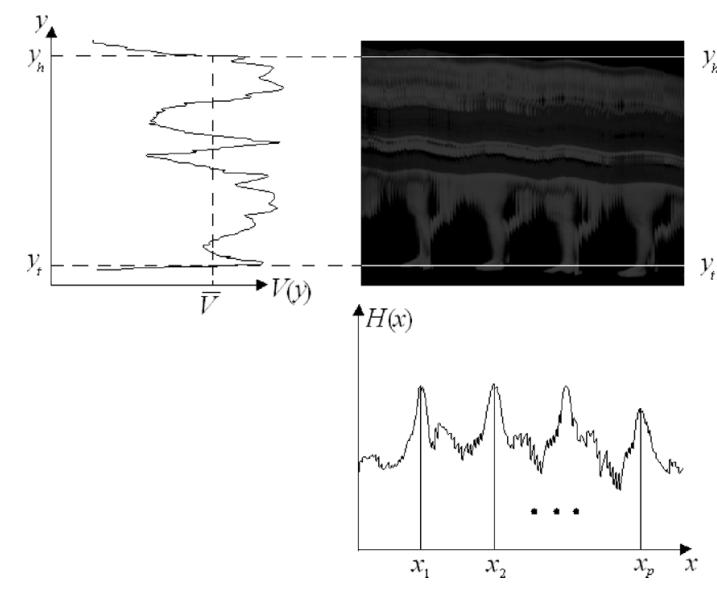

Fig. 3. The horizontal and vertical projections of the walking path image slice.
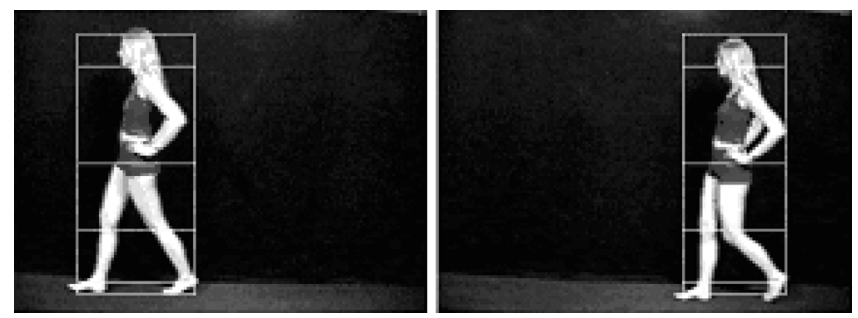

Fig. 4. Sample frames showing the bounding rectangle of the subject complete with body ratio segmentation.

the real positions. However, these bounding rectangles are only an approximation for initializing and terminating the outlining algorithm, reducing computational costs and approximating the segmentation of the body. Imperfect segmentation does not significantly affect the results.

Finally, the average step length is calculated. This is determined by examining the changing width of the subject's bounding rectangle in the video frames. This rectangle broadens and narrows as the walker passes through the periodic phases of gait. The peak width is the walker's step length. By identifying these periodic peaks and obtaining their mean, the average step length, $S$, is obtained.

\section{Outlining}

At ankle height (determined in the bounding rectangle phase), an xt slice is obtained. An initial approximation of the snake is fitted to the braids by searching this image for peaks along the direction of the walking path but at a distance of half the step length from this path in either direction. Once these peak points are found, they are connected together to form the initial zigzagshaped template. This initial template is then warped to follow the pattern's edges using snakes [19]. Because of the similarity of the braided patterns at locally connected slices, snake fitting is initialized only once. After that, the process is repeated at each slice from ankle to head using the result of the snake fitting from the previous to initialize the next. This results in a complete outline of the tracked region throughout the video sequence (see Fig. 5).

\section{E. Ellipse Fitting}

Once the outline of the area being tracked has been obtained and the body has been segmented, the outline segments are used 


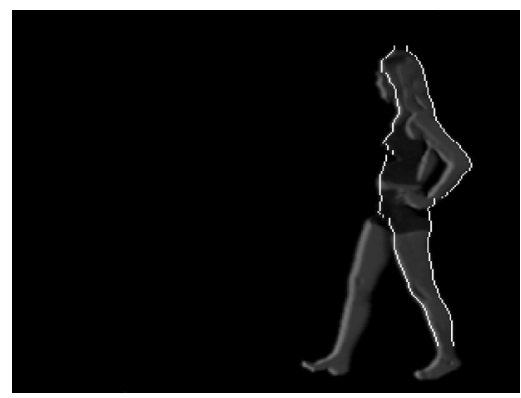

Fig. 5. The outline of the area of interest in a sample frame.

as inputs for the ellipse-fitting algorithm. The outline obtained from the slice-by-slice snake algorithm is divided, using segmentation, into the tracked body parts: head, torso, thigh and shank. An ellipse is attached to each of these parts independently.

Body parts in each frame have now been positioned independent of each other and of their locations in previous frames. This could cause anomalies in results. In many human motiontracking algorithms, segments are positioned subject to constraints and each depends on the location of its predecessor in the hierarchical tree structure of the body. This is a good way of avoiding unlikely positioning but is prone to straying. One bad fit would propagate through the image and through the image sequence causing tracking to fail.

This algorithm rarely suffers from straying and recovers quickly when it does, but can potentially result in nonsensical conclusions. Using our direct ellipse-fitting method, the result will be the best-fit ellipse with no limitations. While this gives a good estimate of the position and orientation of the body part, the size of the part may vary from frame to frame and the relative angles with other body parts could be unreasonable.

To overcome this, constraints are applied after the initial approximate fit. Firstly, the dimensions of each ellipse are set to the average over the sequence, on the basis that changes should not occur. Next, the angles of the ellipses are temporally smoothed using a 1-D Gaussian filter. This ensures that body parts are not rotating at unreasonable speeds from frame to frame and it improves gait graphs. Lastly, relative angles are checked to ensure that joint angles are reasonable.

The final stage of system design is extraction of gait data from the tracked body model. While visual results clearly show the success of the algorithm, the purpose of a gait analysis system is to accurately measure human walking with respect to certain standardized benchmarks. Various useful data can be extracted from the output of this algorithm but for the purposes of validation, sagittal view gait graphs and some significant gait parameters are presented here.

\section{RESULTS}

\section{A. System}

The system was implemented on a PC with 2.66-GHz Pentium 4 processor and $1 \mathrm{~GB}$ of RAM. The implementation was coded in Microsoft Visual $\mathrm{C}++\mathrm{v} 6.0$ and results graphed in MATLAB. Test videos used are $320 \times 240$, 24-bit RGB AVI.
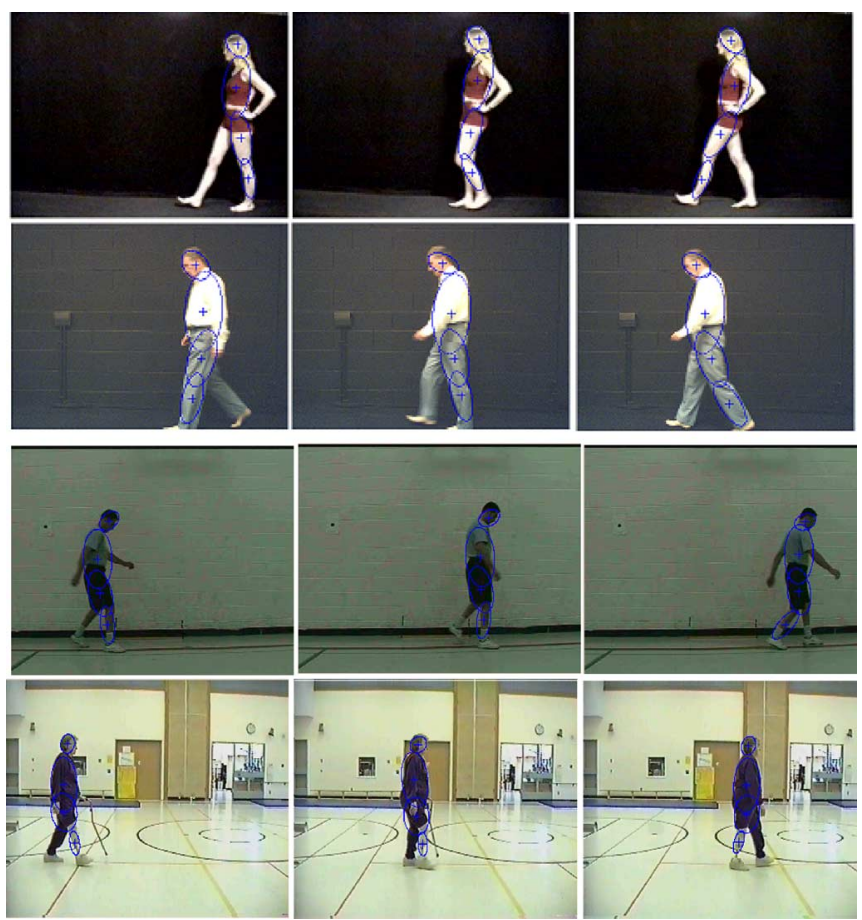

Fig. 6. Sample frames from clips 1-4, showing the simple ellipse model attached to the images of the subjects.

Higher resolution is possible. The sequences are all approximately four seconds long ( 120 frames). Processing time is less than one minute per clip.

Clip 1 was filmed in a gait laboratory using a high quality camcorder and captured uncompressed at 30 frames per second using an ATI All-in-Wonder 128 Pro video capture card. The subject was wearing fitted sportswear with her legs mostly bare and walking without arm swing. This clip was used to perform preliminary tests and also to test the accuracy that could potentially be achieved given minimal obstacles.

Clip 2 was filmed with minimal restrictions placed on the subject's appearance and motion, i.e., the subject is wearing normal clothes and walking naturally with arm swing. This video was filmed with a USB2.0 webcam at a low frame rate ( 15 frames per second) in a reasonably uncontrolled environment, although the background was kept dark to ensure reasonable contrast. The video file was uncompressed. Picture quality and lighting are poor, thus testing the system's sensitivity to these issues. The low frame rate tests the system's ability to interpolate in the presence of missing data.

Clip 3 shows a subject with pathological gait. The frame rate is 30 frames per second and the file was compressed using Indeo video five compression. The background is reasonably homogeneous in this clip, but loose clothing and poor lighting make it difficult to see the outline of the subject's leg throughout. This clip tests the system's ability to measure pathological gait under difficult conditions.

Clip 4 shows a subject with a prosthetic limb using a crutch and walking in a cluttered environment. The frame rate for this clip is 15 frames per second and the file was compressed with IR32 compression. This clip is the toughest test for the system. Difficulties include lighting, contrast, clothing, arm swing, 

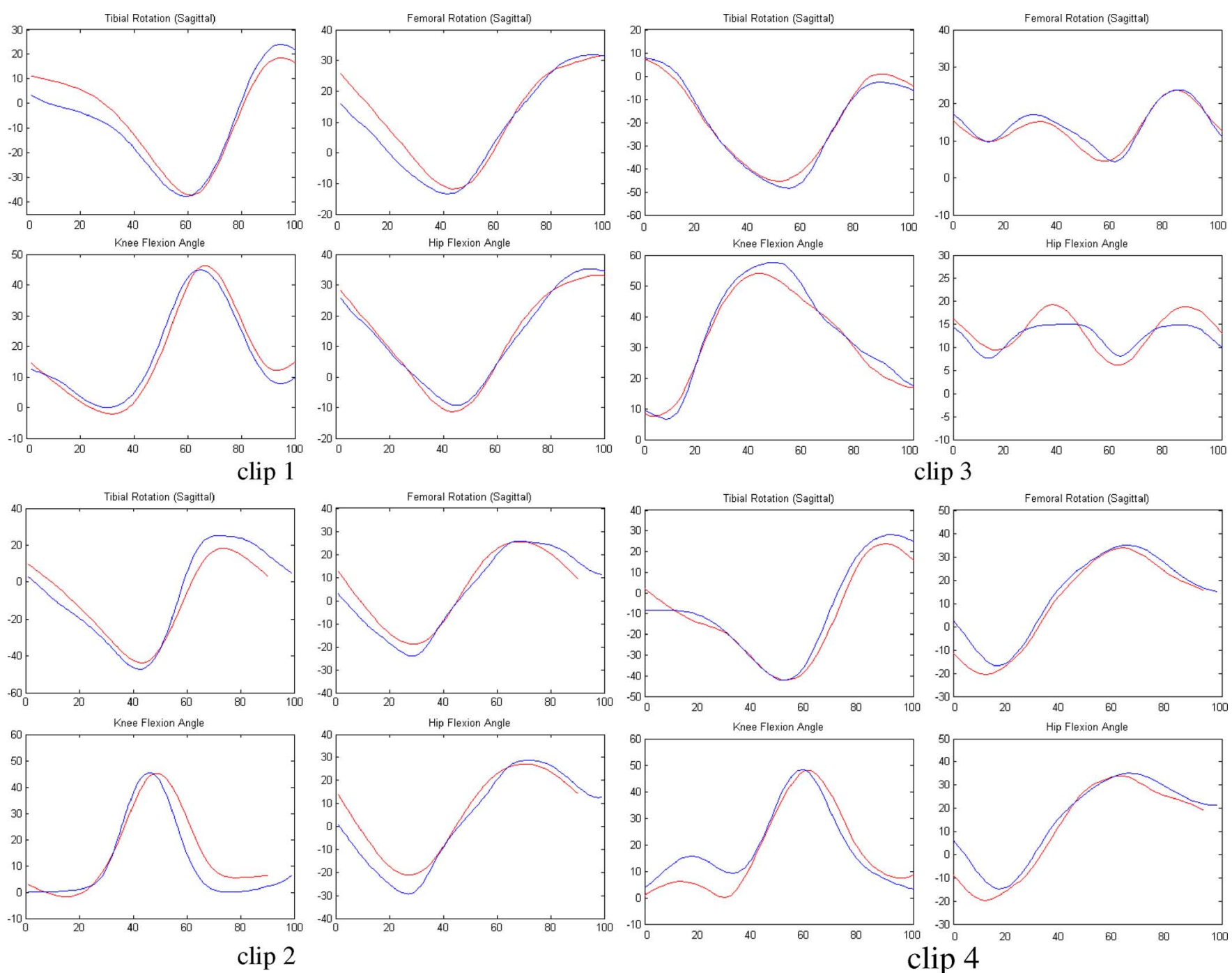

Fig. 7. Gait data acquired from clips $1-4$ plotted alongside manually acquired data.

frame rate, background clutter, nonstandard limb shapes and walking aids.

In order to test the results of this automated system, a program was designed to measure limb segment orientations manually in which the user approximates locations of hip, knee and ankle. Limb segment orientations are calculated from these approximations and saved to a data file. Outputs are then graphed alongside system output for comparison. This method is similar to that used in [20].

\section{B. Visual Results}

Visual data are useful for gauging the success of the algorithm and could be used to create an avatar to mimic gait in a virtual environment. This is a very tangible form of output but is only fully realizable with complete 3-D gait data, i.e., including transverse and coronal planes of movement and pelvis and ankle data. Here, focus is on the sagittal plane and particularly on the main lower limb area, as this is the most challenging region in the acquisition of gait information.

Fig. 6 shows frames from the four video sequences with the ellipse body model attached. Despite significant differences in sequence quality and contrast, there is little difference in the accuracy of the model fitting. However, because the leg is not directly visible in the second sequence, there is an unavoidable ambiguity with regard to the dimensions of the limb segments. In the graphical results, the orientation of the segments throughout the sequence is plotted and so this ambiguity does not affect gait measurements - another advantage of this ellipsebased method.

\section{Gait Data}

As this system concentrates on the main lower limb sections, graphs are presented for rotation of thigh and shank. All angles are relative to the global coordinate system but could be easily adapted to use embedded coordinates. These graphs are typical of gait kinematics. Results shown in Fig. 7 are presented from foot-contact to foot-contact.

In order to test these results, they are compared with manually approximated angles. Because of poor picture quality in some of sequences, it can be difficult to determine the orientation of some segments and so the manual approximations sometimes introduce their own inaccuracies. Manually obtained data are graphed in red. As can be seen, the system output remains close to the manual results. Except in the pathological case, Clip 3, 


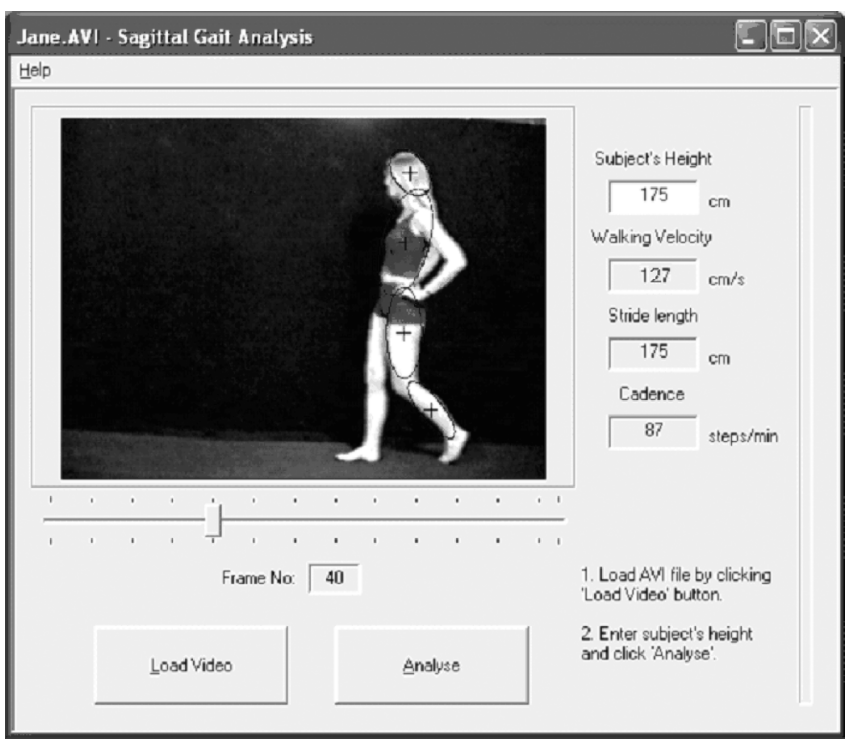

Fig. 8. Simple user-friendly interface.

TABLE I

Gait Parameters AcQuired From the Four Video SeQuences

\begin{tabular}{|l|c|c|c|c|r|}
\hline & \multicolumn{4}{|c|}{ Clip number: } & \multirow{2}{*}{} \\
\cline { 2 - 5 } & $\mathbf{1}$ & $\mathbf{2}$ & $\mathbf{3}$ & $\mathbf{4}$ & \\
\hline Subject's Height & 175 & 180 & 185 & 166 & $\mathrm{~cm}$ \\
\hline Walking Velocity & 127 & 136 & 131 & 141 & $\mathrm{~cm} / \mathrm{sec}$ \\
\hline Stride Length & 175 & 170 & 191 & 133 & $\mathrm{~cm}$ \\
\hline Cadence & 87 & 96 & 82 & 127 & steps/min \\
\hline
\end{tabular}

all outputs exhibit expected shapes and ranges for all graphs. The pathological gait shows stiffness in the hip. Although more exaggerated in the manual results, the same diagnosis would be made from either graph. In clip 2, low frame rate has caused information loss, seen in the stance phase (0\%-30\% of the gait cycle) where the typically sinusoidal shape of the knee flexion graph is lost. The same error occurs in the manually obtained results. In clip 4, the measurement system performs better than the manual method, as it provides smoother outputs allowing the shape to be more easily recognized.

A simple user interface has been designed and demonstrates the ease of use of the system. This is seen in Fig. 8. Along with visual and graphical results, significant gait parameters are normally acquired. These are walking velocity, stride length and cadence. The values for the sample video sequences are presented in Table I.

\section{DISCUSSION}

Few commercial marker-free systems are in current use in gait analysis. They are becoming more common in areas such as sports science, animation and surveillance but the accuracy and detail required for gait analysis makes system design challenging. The greatest obstacles to visual systems such as marker-free systems lie in sagittal-plane acquisition. Thus, this work focuses on designing a reliable marker-free system for monitoring sagittal-plane movement in the gait cycle.

The system was tested on four different video clips made under very different conditions. In all cases, the system compares well with manual measurements and with other published results for equivalent systems, e.g., [21]. While our system shows the potential of an automated marker-free system, a number of improvements have been identified. In the future, the snake algorithm will incorporate learning so that it can automatically set its parameters. Also, 3-D position extraction would also add robustness and accuracy to the measurement and avoid inaccuracies introduced by straying from the frontoparallel path.

This system will allow patients' gait to be recorded in a relaxed and convenient environment without the need for a trained therapist to be present. Thus, therapists can use their expertise to diagnose and treat gait rather than spending time mastering and using marker systems. This system will be used in the $\mathrm{Na}-$ tional Rehabilitation Hospital, Dublin where it was developed, and will become part of a more complete gait laboratory design in the future.

\section{ACKNOWLEDGMENT}

The authors would like to acknowledge the National Rehabilitation Hospital, Dún Laoghaire, Co. Dublin, Ireland for support and facilities. The authors would also like to thank Dr. E. Lemaire of The Rehabilitation Centre in The Ottawa Hospital and S. Nyogi of the Massachusetts Institute of Technology.

\section{REFERENCES}

[1] D. H. Sutherland, "The evolution of clinical gait analysis Part III-Kinetics and energy assessment," Gait Posture, vol. 21, pp. 447-461, 2005.

[2] J. R. W. Morris and A. Macleod, "An investigation of the sources and characteristics of noise in a video-based kinematic measurement system," Models, Connection With Experimental Apparatus and Relevant DSP Techniques for Functional Movement Analysis, Deliverable F, CAMARC (A1012) CEC/AIM Project, 1990.

[3] R. Baker, "Gait analysis methods in rehabilitation," J. Neuroeng. Rehabil., vol. 3, p. 4, 2006.

[4] R. Frischholz and T. Wittenberg, "Computer aided visual motion analysis," Mikromak GmbH, 2001.

[5] A. M. Sabatini, "Real-time Kalman filter applied to biomechanical data for state estimation and numerical differentiation," Med. Biol. Eng. Comput., vol. 41, pp. 2-10, 2003.

[6] R. F. Weir and D. S. Childress, "A portable, real-time, clinical gait velocity analysis system," IEEE Trans. Rehabil. Eng., vol. 5, no. 4, pp. 310-321, Dec. 1997.

[7] A. M. Chaudhari, R. W. Bragg, E. J. Alexander, and T. P. Andriacchi, "A video-based, markerless motion tracking system for biomechanical analysis in an arbitrary environment," ASME BED, vol. 50, pp. 777-778, 2001.

[8] J. Segen, "A camera-based system for tracking people in real time," in Proc. 13th Int. Conf. Pattern Recognit., 1996, vol. 3, pp. 63-67.

[9] J. K. Aggarwal and Q. Cai, "Human motion analysis: A review," in Proc. IEEE Nonrigid Articulated Motion Workshop, 1997, pp. 90-102.

[10] T. B. Moeslund, A. Hilton, and V. Krüger, "A survey of advances in vision-based human motion capture and analysis," Comput. Vis. Image Understand., vol. 104, pp. 90-126, 2006.

[11] J. Courtney, D. P. Burke, and A. M. de Paor, "Application of digital image processing to marker-free analysis of human gait," Measurement Sci. Rev., vol. 1, pp. 11-14, 2001.

[12] K. Rohr and A. K. Systeme, "Human movement analysis based on explicit motion models," in Motion-Based Recognition, M. Shah and R. Jain, Eds. Boston, MA: Kluwer, 1997.

[13] J. Yamato, J. Ohya, and K. Ishii, "Recognizing human action in timesequential images using hidden Markov model," in IEEE Comput. Soc. Conf. Comput. Vis. Pattern Recognit., 1992, pp. 379-385.

[14] A. Blake and M. Isard, "The CONDENSATION algorithm-Conditional density propagation and applications to visual tracking," Adv. Neural Inf. Process. Syst., vol. 9, p. 361, 1997.

[15] J. Deutscher, A. Blake, and I. Reid, "Articulated body motion capture by annealed particle filtering," in IEEE Conf. Comput. Vis. Pattern Recognit., 2000, vol. 2, pp. 126-133. 
[16] J. Courtney and A. M. de Paor, "Direct least-squares ellipse fitting," presented at the 7th IASTED Int. Conf. Computer Graphics Imag., Kauai, HI, 2004.

[17] S. A. Niyogi and E. H. Adelson, "Analyzing and recognizing walking figures in XYT," in Proc. IEEE Comput. Soc. Conf. Comput. Vis. Pattern Recognit. (CVPR), 1994, pp. 469-474.

[18] S. A. Niyogi and E. H. Adelson, "Analyzing gait with spatiotemporal surfaces," in Proc. 1994 IEEE Workshop Motion of Non-Rigid Articulated Objects, 1994, pp. 64-69.

[19] M. Kass, A. Witkin, and D. Terzopoulos, "Snakes: Active contour models," Int. J. Comput. Vis., vol. 1, pp. 321-331, 1988.

[20] E. Lemaire, Motion Analysis Tools [Online]. Available: http://www. irrd.ca/cag/mat/

[21] S. Simcox, S. Parker, G. M. Davis, R. W. Smith, and J. W. Middleton, "Performance of orientation sensors for use with a functional electrical stimulation mobility system," J. Biomechan., vol. 38, pp. 1185-1190, 2005.

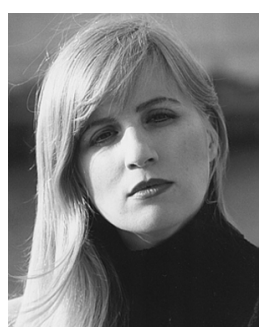

Jane Courtney (M'05) received the B.E., M.Eng.Sc., and Ph.D. degrees from University College Dublin, in 1998, 2001, and 2005, respectively.

She worked as a project engineer in ESBI and as a lecturer in University College Dublin before accepting a position as a full time lecturer in 2004 in the Dublin Institute of Technology (DIT) where she works today. Her research and teaching interests include computer vision, image processing, and power systems engineering. She is part of the Health Engineering and Rehabilitation Team (HEaRT) and the People Oriented Technology (TeaPOT) research groups in DIT and an adjunct member of the Vision Systems Group in Dublin City University.

Dr. Courtney is a member of Engineers Ireland.

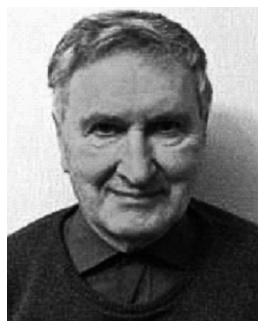

A. M. de Paor was born in Waterford, Ireland, on August 5, 1940. He received the B.E., Ph.D., and D.Sc. degrees in electrical engineering from the National University of Ireland, Dublin, in 1961, 1967, and 1974, respectively. He received the M.S. degree in electrical engineering from the University of California at Berkeley, in 1963.

He lectured at the University of Salford, U.K., from 1963 to 1967, and the National University of Ireland, Dublin, from 1967 to 1969. In 1969, he was appointed Professor of Control Engineering at the University of Salford and on January 1, 1978 became Professor of Electrical Engineering at the National University of Ireland, Dublin. He has been Professor Emeritus since August 31, 2005, but remains active in teaching and research. He has published in control theory, biomedical engineering, renewable energy systems, classical electrical engineering, geomagnetism, and engineering education. Since 1976, he has been a regular visitor at several Universities in Slovakia.

Prof. de Paor is a member of the Royal Irish Academy and a fellow of the Institution of Engineers of Ireland, the Institution of Engineering and Technology, and the Institute of Mathematics and its Applications. 